\title{
Satellite holes of dye molecules doped in polymer films: intermolecular hydrogen-bond effect
}

\author{
Chien-Chih Chiang a,b, Jiann-Hua Wang a, Ji-Yen Cheng a,b, Ta-Chau Chang ${ }^{\text {a, * }}$ \\ a Institute of Atomic and Molecular Sciences, Academia Sinica, P.O. Box 23-166, Taipei 10764, Taiwan, ROC \\ ${ }^{\mathrm{b}}$ Department of Chemistry, National Taiwan University, Taipei, Taiwan, ROC \\ Received 17 January 1995; in final form 13 March 1995
}

\begin{abstract}
We have studied the nonphotochemical hole-burned spectra of dye molecules in polyvinyl alcohol films, where the dye molecule contains a $\mathrm{BF}_{2}$ functional group. An intense satellite hole that possibly corresponds to $\mathrm{B}-\mathrm{F}$ bending is observed. The excitation into the B-F vibronic transition makes the rearrangement of the configurations of the host more efficient via the intermolecular hydrogen bond. In addition, temperature-annealing-cycling results show that the same magnitude of the electron-phonon coupling determines the electronic zero phonon hole (ZPH) and the vibronic satellite hole (SH), even in the presence of the intermolecular hydrogen bond. The hole intensity ratio of the SH to the ZPH suggests that the lowest absorption band consists of at least two prominent absorption components.
\end{abstract}

\section{Introduction}

In amorphous glasses, the absorption bandwidth of a chromophore can be as large as a few hundred wavenumbers due to the inhomogeneity of the local environment, even at low temperature. The spectral hole-burning (HB) method eliminates the inhomogeneous broadening and yields high-resolution siteselective spectra [1-6]. In addition, when tuning the excitation into congested vibronic absorption regions, HB spectra can provide the excited vibrational frequencies of the Franck-Condon active modes [713]. Gorokhovski and Kikas [7] have determined the

\footnotetext{
* Corresponding author.
}

width of the inhomogeneous vibrational distribution from the widths of the ZPH and the SH. Friedrich and Haarer [10] have studied the correlation for the various vibronic transitions to the electronic origin based on their hole widths. They observed that the vibrational inhomogeneity is not uniform but varies with the vibrational modes. Gillie and co-workers [12] have determined the Franck-Condon (FC) factors for the linear electron-vibration coupling of photosystem I. The maximum FC factor of the linear electron-vibration coupling for all modes is $\approx 0.04$. They further found that the energy transport within the antenna is mediated by the phonon and not the intramolecular modes. In addition, they suggested that more than one absorption component is present in the lowest absorption band. 
The linear electron-phonon coupling of the $\mathrm{ZPH}$ has been extensively studied elsewhere [1-6,12-18]. However, to our knowledge, no experimental measurement of the linear electron-phonon coupling of the satellite holes has been reported. As a matter of fact, it is difficult to measure the hole intensity ratio of the phonon sideband hole to its satellite hole because of the weak signal to noise and the interferences from antihole and other satellite holes. In addition, this ratio depends upon burning time and burning power. Recently, we have illustrated that temperature-annealing-cycling (TAC) hole spectra can provide information on the linear electron-phonon coupling [16-18]. The TAC hole spectra are taken as follows: a hole-burned (HB) spectrum is obtained at burning temperature $\left(T_{\mathrm{b}}\right)$ after a hole is produced; then the temperature is raised to the annealing temperature $\left(T_{\mathrm{a}}\right)$, and the annealing spectrum is taken at $T_{\mathrm{a}}$; subsequently, the temperature is cooled back to the cycling temperature $\left(T_{\mathrm{c}}\right)$, and the cycling spectrum is recorded at $T_{\mathrm{c}}$. It is noted that $T_{\mathrm{c}}=T_{\mathrm{b}}$. The hole intensity ratio between annealing and cycling spectra as a function of temperature gives the linear electron-phonon coupling.

Chang and co-workers [18] have applied the TAC method to study how chemical binding of a chromophore to a large group of an oligonucleotide can affect the chromophore-glass interaction. They observed a weak satellite hole in both free chromophore and chromophore-oligonucleotide doped in polyvinyl alcohol (PVOH). The study of the satellite hole is important because it provides information about the local geometry of the chromophore. Small and co-workers [19] found that the hole formation is more efficient in glasses with a hydrogen-bond network. It is important to examine whether the hydrogen-bond effect can enhance the satellite holes, where the vibrational modes are closely related to the hydrogen bond. In this work, we focus on the system 4,4-difluoro-5-(4-phenyl-1,3-butadienyl)-4bora-3a,4a-diaza-s-indacene-3-propionic acid, succinimidyl ester (BODIPY) doped in polyvinyl alcohol (PVOH) films. The BODIPY molecule contains a $\mathrm{BF}_{2}$ functional group, the strong electronegativity of the $\mathrm{F}$ atom can possibly form a hydrogen bond with PVOH. We further apply the TAC method to examine whether the intermolecular hydrogen bond can affect the electron-phonon coupling of a satellite hole relative to that of the $\mathrm{ZPH}$. In addition, an antihole located between the ZPH and the $422 \mathrm{~cm}^{-1}$ $\mathrm{SH}$ is discussed.

\section{Experimental}

In the HB experiments, a home-made sync pump dye laser pumped by an accousto-optically modelocked and $Q$-switched Nd:YAG laser was utilized to produce holes [20]. Our dye laser emitted trains of $\approx 10$ pulses separated by $13 \mathrm{~ns}$ at a repetition rate of $500 \mathrm{~Hz}$. The pulse width was $\approx 35$ ps measured by the autocorrelation function. The fwhm of this dye laser was $\leqslant 2 \mathrm{~cm}^{-1}$ and the wavelength was tunable by tuning a dense flint glass prism and a $100 \mu \mathrm{m}$ étalon in the laser cavity. The absorption spectra were obtained by dispersing the output of a xenon arc lamp (Oriel 68742) through a $1 \mathrm{~m}$ Jobin-Yvon THR 1000 spectrometer with the resolution of $\approx 0.3$ $\AA$ and then splitting the output into two beams. One beam was used to produce a reference signal and the other beam was focused onto the sample to produce a sample signal. The logarithmic ratio of these two signals provides the sample absorbance. The holeburned spectrum was obtained from the difference between the preburn and the postburn spectra. The spot size of the burning beam was $\approx 1 \mathrm{~mm}$ and that of the reading beam was $\approx 200 \mu \mathrm{m}$. Extreme care was taken to ensure that the cross section of the probe beam at the sample was inside the area of the burn laser.

The dye molecule 4,4-difluoro-5-(4-phenyl-1,3butadienyl)-4-bora-3a,4a-diaza-s-indacene-3-propionic acid, succinimidyl ester (BODIPY-2228, Molecular Probes Inc.) was introduced into the polymer solution, which was made by dissolving polyvinyl alcohol (PVOH) in purified deionized water [16]. The syrupy solution was poured onto a quartz plate. The samples were placed in a desiccator with flowing nitrogen gas and were allowed to dry into films. Sample thicknesses were $65 \pm 10 \mu \mathrm{m}$. The samples were mounted on a brass plate with several drilled holes of $\approx 1 \mathrm{~mm}$ diameter and put into a Janis dewar, where they were cooled by cold helium gas. The temperature was measured by a callibrated silicon diode temperature sensor (Lake Shore Cryotronics model). Because of the low thermal conductivity 
of the polymer samples, all spectra were taken after the temperature was stabilized for at least half an hour to ensure the same temperature of the sample as the mount. The deviation of the temperature is \pm 0.5 $\mathrm{K}$ at low temperature.

\section{Results and discussion}

Fig. 1a presents the structure of the BODIPY molecule. Fig. 1b shows the preburn and the postburn hole spectra of BODIPY doped in PVOH at $T_{\mathrm{B}}=6 \mathrm{~K}$. The difference between them gives the hole-burned spectrum as shown in Fig. 1c. The burning wavelength is located at $590 \mathrm{~nm}$ with a photon energy of $2.6 \mu \mathrm{J}$ and a burning time of 15 min. A series of satellite holes to the red of the ZPH appears in Fig. 1. The occurence of SHs is attributed to the excitation into the congested vibronic absorption regions because the inhomogeneous bandwidth is larger than the excited-state vibrational frequencies. Thus, the displacement of the SHs provides the excited-state frequencies of the Franck-Condon active modes [7-13]. Several prominent SHs in Fig. 1b are labeled by their excited-state vibrational frequencies. However, the most striking feature in Fig. $1 \mathrm{~b}$ is the relative hole intensity of the ZPH and the 422 $\mathrm{cm}^{-1} \mathrm{SH}$. It is important to examine why the 422 $\mathrm{cm}^{-1} \mathrm{SH}$ is the most intense one.

Small and co-workers [19] found that the hole formation is more efficient in glasses with a hydrogen-bond network. The strongly electronegative $\mathrm{F}$ atom can possibly form a hydrogen bond with $\mathrm{PVOH}$. Also considering the bending frequency of the $\mathrm{BF}_{3}$ molecule at $\approx 440 \mathrm{~cm}^{-1}$ [21], we propose that the intense hole depth of the $422 \mathrm{~cm}^{-1} \mathrm{SH}$ corresponds to the bending frequency of a $\mathrm{B}-\mathrm{F}$ bond in the BODIPY molecule. The intermolecular hydrogen bond makes the configuration changes of $\mathrm{PVOH}$ more efficient while exciting the BODIPY molecule. This conjecture is further supported by the previous study of the 5-(and 6-)carboxy-X-rhodamine N-hydroxy succinimidyl ester (RhSE) doped in PVOH [18]. No satellite holes around $422 \mathrm{~cm}^{-1}$, to the red of the $\mathrm{ZPH}$, were found in RhSE/PVOH. In addition, a preliminary study on the hole burned spectra of 4,4-difluoro-5-(2-thienyl)-4-bora-3a,4a-diaza-sindacene-3-propionic acid, succinimidyl ester
(BODIPY-2219, Molecular Probe Inc.), a derivative of BODIPY-2228, doped in amorphous glass made from a glycerol-water mixture, is shown in Fig. 2. The burning wavelength is tuned at $555 \mathrm{~nm}$ with a photon energy of $0.09 \mu \mathrm{J}$ and burning time of 60 min. The structure of BODIPY-2219 is also presented in Fig. 2. An intense SH located at $\approx 430$ $\mathrm{cm}^{-1}$ is observed in Fig. 2. The ratios of the optical density changes to the optical densities of the preburn spectra of the ZPH and the $430 \mathrm{~cm}^{-1} \mathrm{SH}$ are $\approx 10 \%$ and $\approx 15 \%$ measured from Fig. 2 , respectively. Considering the structure of these dye molecules, it is suggested that the intense $\mathrm{SH}$ at 422 $\mathrm{cm}^{-1}$ in BODIPY/PVOH must be related to the $\mathrm{BF}_{2}$ functional group of the BODIPY derivatives. The intense SH implies that the intermolecular hydrogen bond could enhance the hole burning efficiency of the specific SHs.

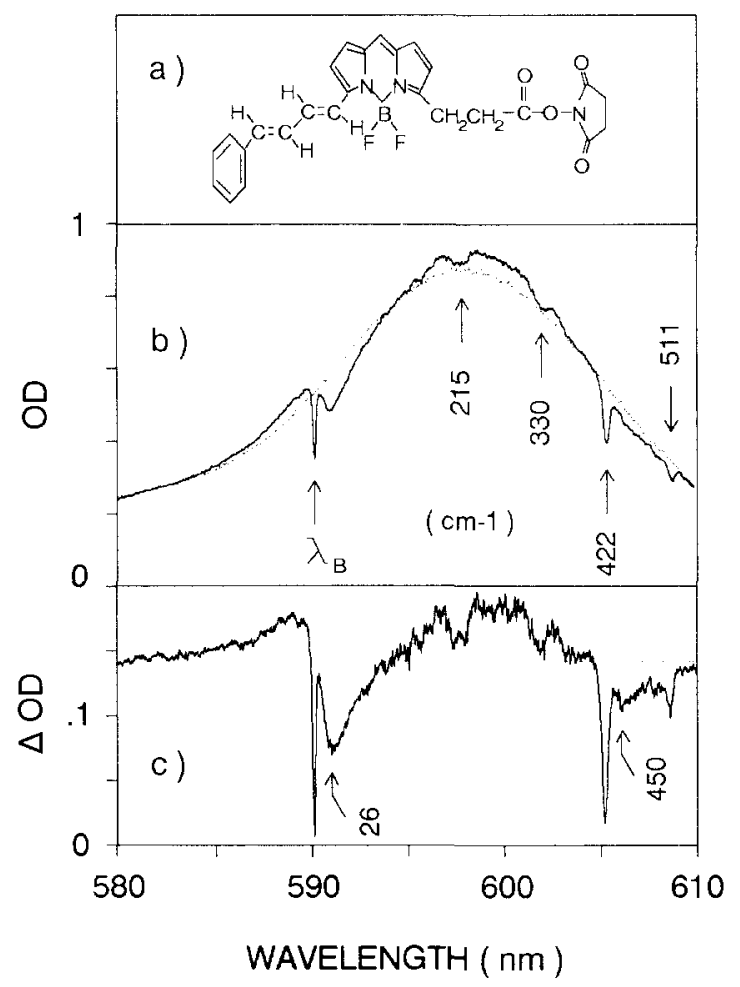

Fig. 1. Hole-burned spectra of BODIPY/PVOH. (a) The structure of BODIPY molecule, (b) the preburn and postburn spectra, and (c) the hole-burned spectrum obtained from the difference between preburn and postburn spectra. Several SHs are labelled with excited-state vibrational frequencies. The burning wavelength $\lambda_{\mathrm{B}}$ $=590 \mathrm{~nm}$. 
It is known that the relative intensities of the SH to the ZPH are mainly determined by the FranckCondon factor and the population of initial vibrational states $[6,14]$. The Franck-Condon factor can be estimated from the ratio of the hole intensity, including the ZPH and its associated phonon sideband hole (PSBH), of the SH to that of the ZPH $[12,13]$. If we assume that the $450 \mathrm{~cm}^{-1}$ hole is the PSBH associated with the $422 \mathrm{~cm}^{-1} \mathrm{SH}$, the intensity ratio of the PSBH to its ZPH is much larger than that of the PSBH to its $422 \mathrm{~cm}^{-1} \mathrm{SH}$ as shown in Fig. 1c. However, it is normally believed that the integrated intensities of the vibronic ZPHs relative to their own PSBHs are determined by the same Debye-Waller factor as in the case of the original $\mathrm{ZPH}$ $[6,14]$. To our knowledge, no experimental evidence from the non-photochemical hole-burning (NPHB) study of this effect has been reported. It is more interesting to examine whether the intermolecular hydrogen-bond effect can perturb the linear electron-phonon coupling of the vibronic transition relative to that of the electronic origin.

It is known that the PSBHs are a manifestation of the linear electron-phonon coupling. We first con- sider the measurement of the Debye-Waller factor of the $\mathrm{ZPH}$. According to the adiabatic approximations, the intensity of the PSBH is determined by the linear electron-phonon coupling [14]. The coupling strength, $S(T)$, can be measured from the DebyeWaller factor $\alpha(T)$ as

$$
\alpha(T)=\frac{I_{\mathrm{ZPH}}(T)}{I_{\mathrm{ZPH}}(T)+I_{\mathrm{PSHB}}(T)}=\exp [-S(T)],
$$

where $I_{\mathrm{ZPH}}$ and $I_{\mathrm{PSHB}}$ correspond to the integrated intensity of a zero-phonon hole and a phonon sideband hole, respectively. It is known that $\alpha(T)$ reflects the host-guest coupling strength, which is mainly determined by the coupling of the low phonon modes of the matrix to the guest molecule. Fig. 3 shows the spectra as a function of burning time by using a photon energy of $1.5 \mu \mathrm{J}$ at $\lambda_{B}=590 \mathrm{~nm}$. It is clear that the integrated hole area of the PSBH increases significantly over that of the $\mathrm{ZPH}$. The variation in the coupling strength due to the broad variety of local environments causes different absorption strengths for different centers of ZPH [22].

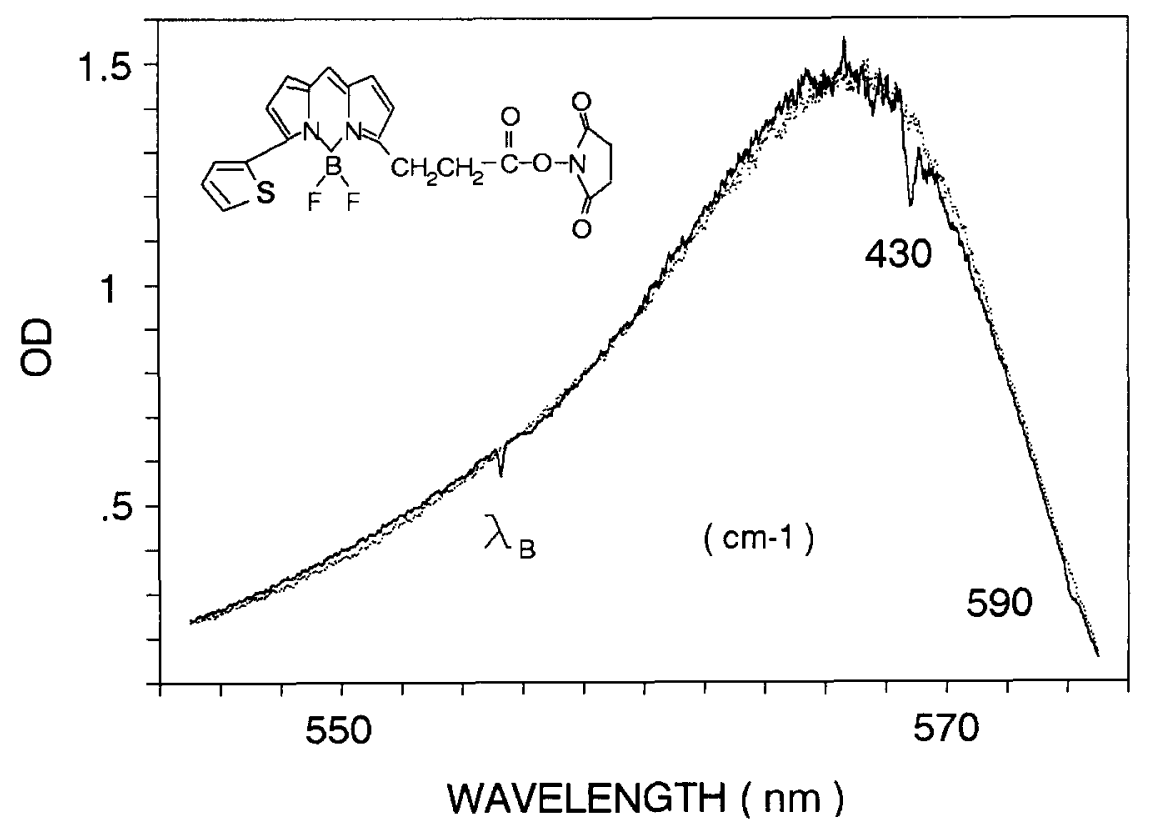

Fig. 2. The preburn and postburn spectra of BODIPY-2219/PVOH. The structure of BODIPY-2219 molecule is shown in the upper corner. Several SHs are labelled with excited-state vibrational frequencies. The burning wavelength $\lambda_{\mathrm{B}}=555 \mathrm{~nm}$. 
Centers with large $\alpha$ values saturate early and centers with small $\alpha$ values saturate much later. Therefore, the different ratios between ZPH and PSBH as a function of burning time are attributed to the different saturation rates. In order to avoid the saturation effect, an extrapolation to zero burning time is necessary [22]. However, the weak signal to noise at short times and the interference from the anti-hole to the $\mathrm{ZPH}$ at relatively long times make the measurement of the Debye-Waller factor difficult as shown in Fig. 3. Similar problems are also exhibited in the measurement of the Debye-Waller factor of the SHs. In addition, the interference from other vibronic transitions to the SH makes the measurement more difficult.

Recently, we have proposed a method to measure the electron-phonon coupling parameter by studying temperature-annealing-cycling (TAC) hole spectra [16-18]. Fig. 4 shows the TAC hole spectra of the $\mathrm{ZPH}$ and its associated $422 \mathrm{~cm}^{-1} \mathrm{SH}$ for the temper-

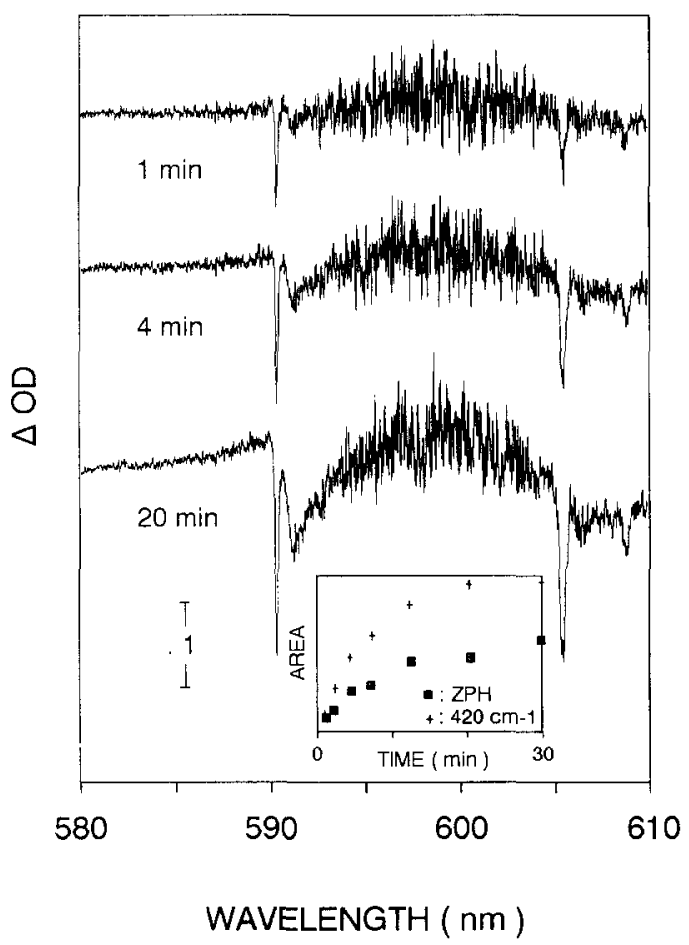

Fig. 3. Time dependent hole-burned spectra of BODIPY/PVOH. The $\triangle O D$ scale is equivalent for all spectra. The inset shows the hole intensities of the ZPH ( $\square)$ and the $422 \mathrm{~cm}^{-1} \mathrm{SH}(+)$ as a function of burning time at $\lambda_{\mathrm{B}}=590 \mathrm{~nm}$. ature cycle of $6 \rightarrow 19 \rightarrow 6 \mathrm{~K}$. The burning time for Fig. 4 is 20 min with a photon energy of $\approx 1.3 \mu \mathrm{J}$. It is observed that both the integrated hole areas of the $\mathrm{ZPH}$ and the $422 \mathrm{~cm}^{-1} \mathrm{SH}$ are reduced when the temperature is raised to $T_{\mathrm{a}}$. However, part of the holes are recovered when the system is cooled back to the burning temperature $T_{c}$. In the temperatureannealing spectra, the reduction of hole area involves temperature-irreversible and temperature-reversible processes. The former process is attributed to the populations of $\Delta N_{j}$ in the product states returning to the initial state. The reversible process is due to the photons absorbed by the thermally activated populations via the linear electron-phonon coupling in the product states, characterized by the asymmetric energies of $\epsilon_{\mathrm{a}} \leqslant k T_{\mathrm{a}}$. In the temperature-cycling spectra, those populations of $\Delta N_{j}$ that returned back to the initial state are temperature-irreversible [23-25], but the thermally activated populations are retrapped since $\epsilon_{\mathrm{a}}>k T_{\mathrm{b}}$. Thus, the difference of hole reduction between annealing and cycling spectra is attributed to the linear electron-phonon coupling [1618].

The theory of linear electron-phonon coupling predicts that the integrated intensity of the $\mathrm{ZPH}$ decreases when temperature increases because of the spectral redistribution of the thermal occupation of phonon states [4,26-29]. The coupling parameter of $S(T)$ as a function of temperature is given by [4]

$S(T)=S_{0}[2 \bar{n}(T)+1]$,

where $S_{0}$ is the coupling constant at $T=0 \mathrm{~K}$ and $\tilde{n}(T)$ are the thermal occupation numbers at the mean phonon frequency of $\omega_{\mathrm{P}}, \tilde{n}(T)=\left[\exp \left(h \omega_{\mathrm{P}} / k_{\mathrm{B}} T\right)-\right.$ $1]^{-1}$. In the annealing HB spectra, the hole is burned at a low temperature, but is probed at higher temperatures. The Debye-Waller factor $\alpha\left(T_{\mathrm{a}}\right)$ follows

$$
\alpha\left(T_{\mathrm{a}}\right)=\exp \left[-S_{0}\left(2 \tilde{n}\left(T_{\mathrm{a}}\right)+2\right)\right],
$$

while $T_{\mathrm{b}} \rightarrow 0 \mathrm{~K}$. However, the hole is burned and probed at the same low temperature in the cycling spectra, where the Debye-Waller factor $\alpha\left(T_{\mathrm{c}}\right)$ follows

$\alpha\left(T_{\mathrm{c}}\right)=\exp \left[-2 S_{0}\right]$ 
Therefore, the ratio of these two intensities $I_{\mathrm{ZPH}}\left(T_{\mathrm{a}}\right) / I_{\mathrm{ZPH}}\left(T_{\mathrm{c}}\right)$ as a function of temperature should follow the relationship

$$
\begin{aligned}
I_{\mathrm{ZPH}}\left(T_{\mathrm{a}}\right) / I_{\mathrm{ZPH}}\left(T_{\mathrm{c}}\right) & =\alpha\left(T_{\mathrm{a}}\right) / \alpha\left(T_{\mathrm{c}}\right) \\
& =\exp \left[-2 S_{0} \tilde{n}\left(T_{\mathrm{a}}\right)\right] .
\end{aligned}
$$

Two variables, $S_{0}$ and $\omega_{\mathrm{P}}$, are used in Eq. (5) to determine the fit to experimental results. The value of $\omega_{\mathrm{P}}=25 \mathrm{~cm}^{-1}$ is measured from the frequency difference between ZPH and PSBH. The solid curve shown in Fig. 5a is calculated from Eq. (5) by using $\omega_{\mathrm{P}}=25 \mathrm{~cm}^{-1}$ and $S_{0}=0.55$. The TAC data of the counterpart of $I_{\mathrm{ZPH}}\left(T_{\mathrm{a}}\right) / I_{\mathrm{ZPH}}\left(T_{\mathrm{c}}\right)$ obtained at $\lambda_{\mathrm{B}}=$ $590 \mathrm{~nm}$ are plotted as squares in Fig. 5a. The agreement between experiment and calculation appears reasonable.

Fig. 4 shows that the changes of hole area in the TAC hole spectra of the $422 \mathrm{~cm}^{-1} \mathrm{SH}$ are similar to that of the ZPH. The counterparts of $I_{\mathrm{SH}}\left(T_{\mathrm{a}}\right) / I_{\mathrm{SH}}\left(T_{\mathrm{c}}\right)$ measured at $\lambda_{\mathrm{B}}=590 \mathrm{~nm}$ are plotted in Fig. $5 \mathrm{~b}$. Using the value of $\omega_{\mathrm{P}}=25 \mathrm{~cm}^{-1}$ and the magnitudes of $S_{0}=0.5$ in Eq. (5), the simulation agrees with the experimental results. Considering the exper- imental errors, this linear electron-phonon coupling parameter agrees with the above measurement of the parameter of the ZPH. To our knowledge, this is the first experimental evidence from NPHB results to illustrate that the relative intensities of the PSBHs to their respective electronic ZPHs and SHs are determined by the same Debye-Waller factor, even in the presence of the hydrogen bond effect. It is suggested that the hydrogen bond between the chromophore and glass does not perturb the linear electron-phonon coupling of the SH and the ZPH differently.

In order to examine whether the hole intensity ratio of the $\mathrm{SH}$ to the $\mathrm{ZPH}$ has a burning time dependence, the inset in Fig. 3 shows the growth of integrated hole intensities of the ZPH and the 422 $\mathrm{cm}^{-1} \mathrm{SH}$ as a function of burning time. The intensity ratio of the $422 \mathrm{~cm}^{-1} \mathrm{SH}$ to the $\mathrm{ZPH}$ is $1.8 \pm 0.2$ after two minutes burning time. Since the absorbance of the preburn spectra at the ZPH and the $422 \mathrm{~cm}^{-1}$ $\mathrm{SH}$ are approximately identical, the scaling factor of the intensity ratio is $\approx 1$. Thus, the ratio of $1.8 \pm 0.2$ implies that the intense $\mathrm{SH}$ of the $422 \mathrm{~cm}^{-1}$ mode cannot be described simply by the FC factor. We

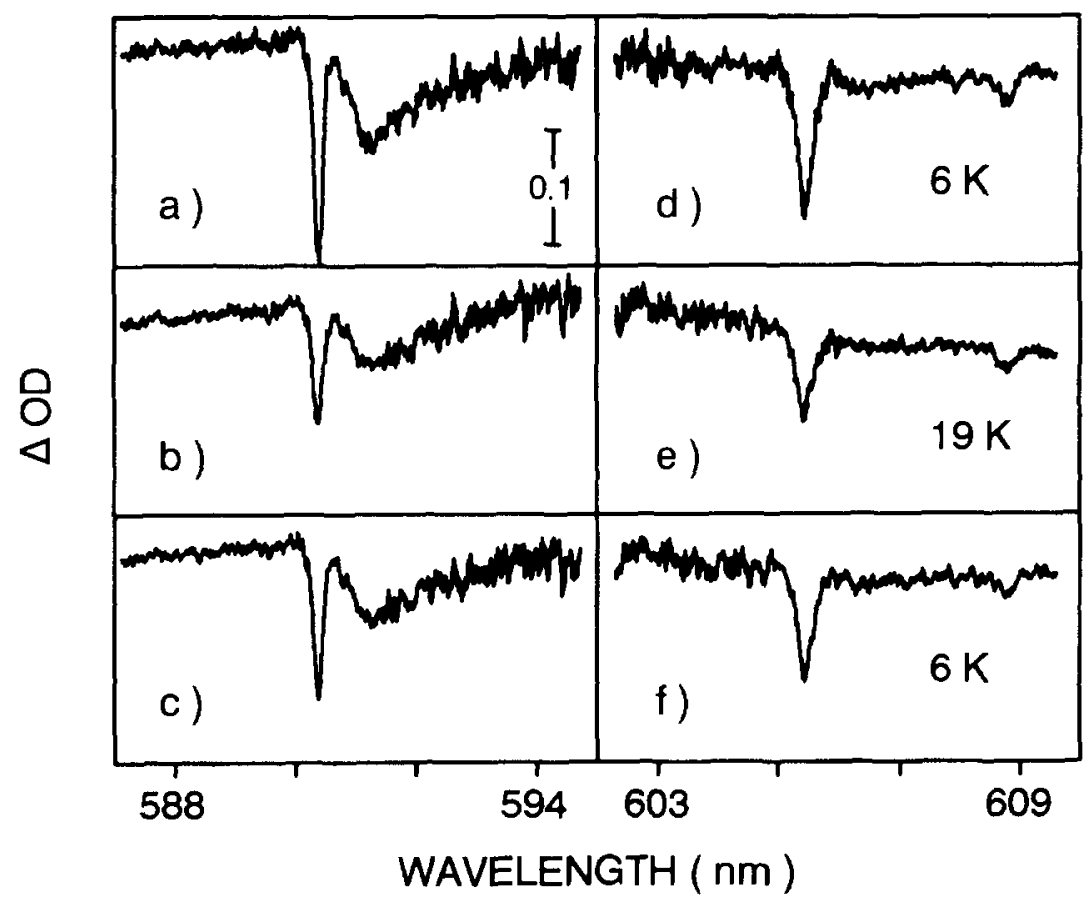

Fig. 4. Typical temperature-annealing-cycling hole spectra of the ZPH (a)-(c) and the $422 \mathrm{~cm}^{-1} \mathrm{SH}$ (d)-(f) of BODIPY/PVOH. 


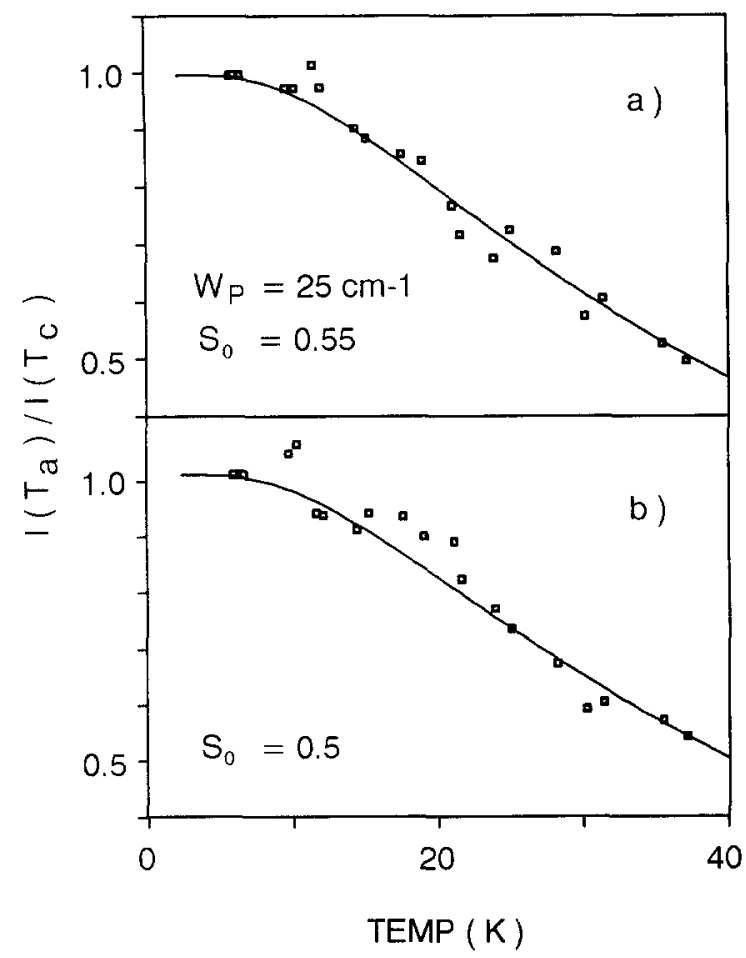

Fig. 5. The plots of the ratio $I_{\mathrm{ZPH}}\left(T_{\mathrm{a}}\right) / I_{\mathrm{ZPH}}\left(T_{\mathrm{c}}\right)$ of the $\mathrm{ZPH}$ (a) and $I_{\mathrm{SH}}\left(T_{\mathrm{a}}\right) / I_{\mathrm{SH}}\left(T_{\mathrm{c}}\right)$ of the $422 \mathrm{~cm}^{-1} \mathrm{SH}(\mathrm{b})$ as a function of annealing temperature. The burning wavelength is located at 590 $\mathrm{nm}$. The solid lines are the fit to the experimental data ( $\square$ ) using Eq. (5).

believe that the lowest absorption band consists of the electronic absorption and the vibronic transition components.

We have further tuned the burning wavelength to the red of the absorption band to determine the FC factor of the $422 \mathrm{~cm}^{-1} \mathrm{SH}$ to the $\mathrm{ZPH}$. We assume that the excitation of the vibronic transition is negligible at $\lambda_{\mathrm{B}}=600 \mathrm{~nm}$. Our experimental results show that the intensity ratio of the $422 \mathrm{~cm}^{-1} \mathrm{SH}$ to the $\mathrm{ZPH}$ is $\approx 0.09$. Considering the scaling factor of the absorbance at the ZPH and $422 \mathrm{~cm}^{-1} \mathrm{SH}$ being $\approx 5$ and their corresponding bandwidths of 4.5 and 8 $\mathrm{cm}^{-1}$, the FC factor of the $422 \mathrm{~cm}^{-1} \mathrm{SH}$ to the $\mathrm{ZPH}$ is estimated to be $0.25 \pm 0.05$.

Another noteworthy feature related to the 422 $\mathrm{cm}^{-1} \mathrm{SH}$ is the antihole structure as shown in Fig. 1c. Besides a broad antihole to the blue side of the $\mathrm{ZPH}$, another broad antihole is located between the $\mathrm{ZPH}$ and the $422 \mathrm{~cm}^{-1} \mathrm{SH}$. The possible mechanism for the blue-shifted antihole of the ZPH is discussed by Shu and Small [30]. They suggested that NPHB occurs as a result of an outside-in hierarchy of tunneling events, triggered by electronic excitation. Consequently, the free volume for the chromophore increases for the postburn configuration. The increase in free volume can explain the blue-shifted antihole since $\pi \pi^{*}$ transitions typically undergo a red-shift from gas to condensed phases. In addition, the compensation of the total intensity of the $\mathrm{ZPH}$ and its PSBH to the intensity of the blue-shifted antihole indicates that the hole production must result from the configurational relaxation of the chromophore-glass induced by excitation [12]. In this work, the excitation of the BODIPY can possibly increase the free volume around the chromophore. Since the vibrational relaxation is much faster than the hole formation, the antihole in the blue side of the $422 \mathrm{~cm}^{-1} \mathrm{SH}$ is very possible due to the excitation of the BODIPY. However, the antihole structure of the $422 \mathrm{~cm}^{-1} \mathrm{SH}$ differs from that of the $\mathrm{ZPH}$. We consider that the excitation of the vibronic transition may be responsible for the difference between the antihole structures of the $422 \mathrm{~cm}^{-1} \mathrm{SH}$ and the $\mathrm{ZPH}$. In addition, with reference to the hole structure of other satellite holes, we believe that the motion of the B-F mode could make an additional effect in the antihole structure of the $422 \mathrm{~cm}^{-1} \mathrm{SH}$.

\section{Concluding remarks}

This work establishes that the intermolecular hydrogen bond can enhance the hole-burning efficiency of the SHs, where the vibrational modes are closely related to the hydrogen bond. Our experimental results suggest that the intense $\mathrm{SH}$ located at $422 \mathrm{~cm}^{-1}$ corresponds to the bending frequency of the B-F bond in the BODIPY molecule. In addition, we have illustrated that the TAC method can provide reliable measurement of the linear electron-phonon coupling for both the electronic and the vibronic transitions. It is confirmed that the same magnitude of the Debye-Waller factor determines the PSBH of the $\mathrm{ZPH}$ and the PSBH of the vibronic-ZPH (SH), even in the presence of intermolecular hydrogen bonding. The $1.8 \pm 0.2$ value of the intensity ratio of the $\mathrm{SH}$ to the $\mathrm{ZPH}$ measured in this work suggests that the 
$590 \mathrm{~nm}$ excitation burns into at least two absorption components. Together with the observation of the antihole associated with the $422 \mathrm{~cm}^{-1} \mathrm{SH}$, we believe these evidences show that a strong vibronic absorption component associated with the $422 \mathrm{~cm}^{-1}$ $\mathrm{SH}$ is present in the lowest absorption band. The prominent structure of the $422 \mathrm{~cm}^{-1} \mathrm{SH}$ is attributed to the intermolecular hydrogen bond between the BODIPY and PVOH.

It is further suggested that the chromophore containing a hydrogen bond should be avoided for the application of frequency domain optical storage. However, this specific property is important to the study of the DNA-chromophore interaction. This is because DNA could form hydrogen bonds with the chromophore, which can significantly perturb the chromophore-glass interaction [31].

\section{Acknowledgement}

This work was supported by the Academia Sinica and the NSC of the Republic of China (Grant NO. NSC-84-2113-M-001-025). The authors wish to thank the referee for useful suggestions.

\section{References}

[1] A.A. Gorokhovskii, R.K. Kaarli and L.A. Rebane, JETP Letters 20 (1974) 216.

[2] G.J. Small, in: Spectroscopy and excitation dynamics of condensed molecular systems, eds. V.M. Agranovich and R.M. Hochstrasser (North-Holland, Amsterdam, 1983).

[3] R.I. Personov, in: Spectroscopy and excitation dynamics of condensed molecular systems, eds. V.M. Agranovich and R.M. Hochstrasser (North-Holland, Amsterdam, 1983).

[4] J. Friedrich and D. Haarer, Angew. Chem. 96 (1984) 96; Angew. Chem. Intern. Ed. Engl. 23 (1984) 113.

[5] J. Friedrich and D. Haarer, in: Optical spectroscopy of glass, ed. I. Zschokke-Gränacher (Reidel, Dordrecht, 1986).
[6] W.E. Moerner, ed., Topics in current physics, Persistent spectral hole burning: science and applications (Springer, Berlin, 1988).

[7] A.A. Gorokhovski and J. Kikas, Opt. Commun. 21 (1977) 272.

[8] B.M. Kharlamov, L.A. Bykovskaya and R.I. Personov, Chem. Phys. Letters 50 (1977) 407.

[9] J.M. Hayes and G.J. Small, Chem. Phys. 27 (1978) 151.

[10] J. Friedrich and D. Haarer, J. Chem. Phys. 79 (1983) 1612.

[11] B.L. Fearey, T.P. Carter and G.J. Small, J. Phys. Chem. 87 (1983) 3590.

[12] J.K. Gillie, G.J. Small and J.H. Golbeck, J. Phys. Chem. 93 (1989) 1620.

[13] N. Murase and K. Horie, J. Chem. Phys. 99 (1993) 6313.

[14] K.K. Rebane, Impurity spectra of solids (Plenum Press, New York, 1970).

[15] W. Breinl, J. Friedrich and D. Haarer, J. Chem. Phys. 80 (1984) 3496; 81 (1984) 3915.

[16] T.-C. Chang, C.C. Chiang, G.J. Small and S.H. Chou, Chem. Phys. Letters 223 (1994) 190.

[17] T.-C. Chang, C.C. Chiang, S.H. Chou and K. Peck, J. Luminescence (1994), in press.

[18] T.-C. Chang, C.C. Chiang, S.H. Chou and K. Peck, J. Phys. Chem. (1994), in press.

[19] B.L. Fearey, R.P. Stout, J.M. Hayes and G.J. Small, J. Chem. Phys. 78 (1983) 7013.

[20] T.-C. Chang, B.H. Jou, R.S. Ou, C.C. Chiang and H.W. Li, Chem. Phys. Letters 187 (1991) 208.

[21] H.S. Booth and D.R. Martin, Boron trifluoride and its derivatives (Wiley, New York, 1949).

[22] J. Friedrich, J.D. Swalen and D. Haarer, J. Chem. Phys. 73 (1980) 705.

[23] W. Köhler, J. Meiler and J. Friedrich, Phys. Rev. B 35 (1987) 4031.

[24] P. Schellenberg, J. Friedrich and E. Daltrozzo, J. Chem. Phys. 95 (1991) 189.

[25] A. Elschner and H. Bässler, J. Luminescence 43 (1989) 33.

[26] M. Born and K. Huang, Dynamics theory of crystal lattice (Oxford Univ. Press, Oxford, 1954).

[27] T.-C. Chang, S.H. Chou, H.W. Li and S.H. Lin, J. Chem. Phys. 99 (1993) 2781.

[28] A. Furusawa, K. Horie and I. Mita, Chem. Phys. Letters 161 (1989) 227.

[29] R. Jankowiak, J.M. Hayes and G.J. Small, Chem. Rev. 93 (1993) 1471.

[30] L. Shu and G.J. Small, Chem. Phys. 141 (1990) 447; J. Opt. Soc. Am. B 9 (1992) 724.

[31] T.-C. Chang, C.C. Chiang and K. Peck, in preparation. 